\title{
First Measurement of the $\rho$ Spectral Function in High-Energy Nuclear Collisions
}

R. Arnaldi, ${ }^{9}$ R. Averbeck,${ }^{8}$ K. Banicz,${ }^{4,2}$ J. Castor, ${ }^{3}$ B. Chaurand,${ }^{6}$ C. Cicalò, ${ }^{1}$ A. Colla, ${ }^{9}$ P. Cortese, ${ }^{9}$ S. Damjanovic, ${ }^{4}$ A. David, ${ }^{5,2}$ A. De Falco, ${ }^{1}$ A. Devaux,${ }^{3}$ A. Drees,${ }^{8}$ L. Ducroux,${ }^{10}$ H. En'yo, ${ }^{7}$ J. Fargeix,${ }^{3}$ A. Ferretti, ${ }^{9}$ M. Floris, ${ }^{1}$ A. Förster, ${ }^{2}$ P. Force, ${ }^{3}$ N. Guettet, ${ }^{2,3}$ A. Guichard,${ }^{10}$ H. Gulkanian, ${ }^{11}$ J. M. Heuser, ${ }^{7}$ M. Keil,${ }^{5,2}$ L. Kluberg,,${ }^{6,2}$ C. Lourenço, ${ }^{2}$ J. Lozano, ${ }^{5}$ F. Manso, ${ }^{3}$ A. Masoni, ${ }^{1}$ P. Martins,${ }^{5,2}$ A. Neves,${ }^{5}$ H. Ohnishi, ${ }^{7}$ C. Oppedisano, ${ }^{9}$ P. Parracho,${ }^{2}$ Ph. Pillot, ${ }^{10}$ G. Puddu, ${ }^{1}$ E. Radermacher, ${ }^{2}$ P. Ramalhete, ${ }^{2}$ P. Rosinsky, ${ }^{2}$ E. Scomparin, ${ }^{9}$ J. Seixas,${ }^{5,2}$ S. Serci, ${ }^{1}$ R. Shahoyan, ${ }^{5,2}$ P. Sonderegger, ${ }^{5}$ H. J. Specht, ${ }^{4,2}$ R. Tieulent, ${ }^{10}$ G. Usai, ${ }^{1}$ R. Veenhof,,${ }^{5,2}$ and H. K. Wöhri ${ }^{5,2}$

(NA60 Collaboration)

\author{
${ }^{1}$ Università di Cagliari and INFN, Cagliari, Italy \\ ${ }^{2}$ CERN, Geneva, Switzerland \\ ${ }^{3} L P C$, Université Blaise Pascal and CNRS-IN2P3, Clermont-Ferrand, France \\ ${ }^{4}$ Universität Heidelberg, Heidelberg, Germany \\ ${ }^{5}$ CFTP, Instituto Superior Técnico, Lisbon, Portugal \\ ${ }^{6}$ LLR, Ecole Polytechnique and CNRS-IN2P3, Palaiseau, France \\ ${ }^{7}$ RIKEN, Wako, Saitama, Japan \\ ${ }^{8}$ SUNY, Stony Brook, New York, USA \\ ${ }^{9}$ Università di Torino and INFN, Turin, Italy \\ ${ }^{10} I P N L$, Université Claude Bernard Lyon-I and CNRS-IN2P3, Villeurbanne, France \\ ${ }^{11}$ YerPhI, Yerevan, Armenia
}

(Received 12 December 2005; published 27 April 2006)

\begin{abstract}
We report on a precision measurement of low-mass muon pairs in $158 \mathrm{AGeV}$ indium-indium collisions at the CERN SPS. A significant excess of pairs is observed above the yield expected from neutral meson decays. The unprecedented sample size of 360000 dimuons and the good mass resolution of about $2 \%$ allow us to isolate the excess by subtraction of the decay sources. The shape of the resulting mass spectrum is consistent with a dominant contribution from $\pi^{+} \pi^{-} \rightarrow \rho \rightarrow \mu^{+} \mu^{-}$annihilation. The associated space-time averaged $\rho$ spectral function shows a strong broadening, but essentially no shift in mass. This may rule out theoretical models linking hadron masses directly to the chiral condensate.
\end{abstract}

PACS numbers: 25.75.- q, 12.38.Mh, 13.85.Qk

According to quantum chromodynamics (QCD), strongly interacting matter will, at sufficiently high temperatures or baryon densities, undergo a phase transition from a state of hadronic constituents to quark matter, a plasma of deconfined quarks and gluons. At the same time, chiral symmetry, spontaneously broken in the hadronic world, will be restored. High-energy nucleus-nucleus collisions provide the only way to investigate this issue in the laboratory.

Among the observables used for the diagnostics of the hot and dense fireball formed in these collisions, lepton pairs are particularly attractive. In contrast to hadrons, they directly probe the entire space-time evolution of the fireball and freely escape from the interaction zone, undisturbed by final-state interactions. In the hadronic phase, thermal dilepton production in the mass region $<1 \mathrm{GeV} / c^{2}$ is largely mediated by the light vector mesons $\rho, \omega$, and $\phi$. Among these, the $\rho\left(770 \mathrm{MeV} / c^{2}\right)$ is the most important, due to its strong coupling to the $\pi \pi$ channel and its lifetime of only $1.3 \mathrm{fm} / c$, much shorter than the lifetime of the fireball. These properties have given it a key role as the test particle for "in-medium modifications" of hadron properties close to the QCD phase boundary. The "dual" role of the $\rho$, probing the medium and having the medium probe its own properties, was pointed out already more than 20 years ago: changes both in width and in mass were suggested as precursor signatures of the chiral transition [1]. There seems to be some consensus now that the width of the $\rho$ should increase towards the transition region, based on a number of quite different theoretical approaches [1-5]. On the other hand, no consensus exists on how the mass of the $\rho$ should change in approaching the transition: predictions exist for a decrease [1,6-8], a constant behavior [4,5], and even an increase [3]. Strongly increasing widths and nearly constant masses are also predicted for pions and for nucleons [9].

Experimentally, low-mass electron pair production was previously investigated at the CERN SPS by the CERES/ NA45 experiment for $p$ - $\mathrm{Be} / \mathrm{Au}, \mathrm{S}-\mathrm{Au}$, and $\mathrm{Pb}-\mathrm{Au}$ collisions [10-12]. The common feature of all results from nuclear collisions was an excess of the observed dilepton yield above the expected electromagnetic decays of neutral mesons, by a factor of $2-3$, for masses above $0.2 \mathrm{GeV} / c^{2}$. The surplus yield has generally been attributed to direct thermal radiation from the fireball, dominated by pion 
annihilation $\pi^{+} \pi^{-} \rightarrow \rho \rightarrow l^{+} l^{-}$with an intermediate $\rho$ which is strongly modified by the medium. Statistical accuracy and mass resolution of the data were, however, not sufficient to reach any sensitivity to the character of the in-medium changes.

In this Letter, we present first results on low-mass muon pair production from the NA60 experiment at the CERN SPS. Compared to the results from CERES, we have been able to improve the statistical accuracy by a factor of $>1000$ and the mass resolution by a factor of $2-3$. This has allowed us, for the first time in this field, to isolate the excess by subtraction of the expected sources. The essential features of the resulting mass spectrum, interpreted as the space-time averaged $\rho$ spectral function associated with pion annihilation, can be summarized in a few words: the $\rho$ strongly broadens, but does not show any shift in mass.

The NA60 apparatus complements the muon spectrometer previously used by NA50 with a high-granularity silicon pixel telescope of unprecedented radiation tolerance $[13,14]$. The telescope, embedded in a $2.5 \mathrm{~T}$ dipole magnet in the vertex region, tracks all charged particles before the hadron absorber and determines their momenta independently of the muon spectrometer. The matching of the muon tracks before and after the hadron absorber, both in angular and momentum space, improves the dimuon mass resolution in the region of the vector mesons $\omega, \phi$ from $\sim 80$ to $\sim 20 \mathrm{MeV} / c^{2}$, significantly reduces the combinatorial background due to $\pi$ and $K$ decays and makes it possible to measure the muon offset with respect to the interaction vertex [15]. The additional bend by the dipole field in the target region deflects soft muons into the acceptance of the muon spectrometer, thereby strongly enhancing the opposite-sign dimuon acceptance at low mass and low transverse momentum with respect to all previous dimuon experiments [16]. The rapidity coverage is $3.3<y<4.3$ for the $\rho$, at low $p_{T}$, compared to $3<y<$ 4 for the $J / \psi$. The acceptance, about $1 \%$ or smaller relative to $4 \pi$ in the whole low-mass region [16], increases with $p_{T}$ by a factor of 3 from 0 to $2 \mathrm{GeV} / c$ for the $\rho$ and, integrated over $p_{T}$, increases with mass by factors of $3-6$ from 0.4 to $0.8 \mathrm{GeV} / c^{2}$, depending on the input $p_{T}$ spectrum. Finally, the selective dimuon trigger and the fast readout speed of the vertex tracker allow the experiment to run at very high luminosities, leading to an unprecedented level of statistics for low-mass lepton pairs.

The results reported in this Letter were obtained from the analysis of data taken in 2003 with a $158 \mathrm{AGeV}$ indium beam, incident on a segmented indium target of seven disks with a total of $18 \%$ (In-In) interaction length. At an average beam intensity of $5 \times 10^{7}$ ions per $5 \mathrm{~s}$ burst, about $3 \times 10^{12}$ ions were delivered to the experiment. A total of $230 \times 10^{6}$ dimuon triggers were recorded on tape, collected with two field settings of the muon-spectrometer magnet; the data reported here correspond to the lower of the two settings.

The data reconstruction starts with the muonspectrometer tracks. Next, pattern recognition and tracking in the vertex telescope are done; the interaction vertex in the target is reconstructed with a resolution of $\sim 200 \mu \mathrm{m}$ for the $z$ coordinate and 10-20 $\mu \mathrm{m}$ in the transverse plane. Only events with one vertex are kept; interaction pileup and reinteractions of secondaries and fragments are rejected. Finally, each muon-spectrometer track is extrapolated to the vertex region and matched to the tracks from the vertex telescope. The matching is done using the square of a weighted distance $\left(\chi^{2}\right)$, in a space of angles and inverse momenta, between the two tracks [15]. Correct matches are statistically distinguished from fake matches (associations of muons to nonmuon vertex tracks) by the very different shape of the matching $\chi^{2}$ distributions. Matched tracks are kept if their $\chi^{2}$ is below a certain threshold. Pair reconstruction efficiencies vary by $<10 \%$ both vs mass and vs centrality.

The combinatorial background of uncorrelated muon pairs mainly originating from $\pi$ and $K$ decays is determined using a mixed-event technique [15]. Two single muons from different like-sign dimuon triggers are combined into muon pairs in such a way as to accurately account for details of the acceptance and trigger conditions. The quality of the mixed-event technique can be judged by comparing the like-sign distributions generated from mixed events with the measured like-sign distributions. It is remarkable that the two agree to within $\sim 1 \%$ over a dynamic range of 4 orders of magnitude in the steeply falling mass spectrum [15]. After subtraction of the combinatorial background, the remaining oppositesign pairs still contain "signal" fake matches, a contribution which is only $7 \%$ of the combinatorial background level. It has been determined in the present analysis by an overlay Monte Carlo method. We have verified that an event-mixing technique gives the same results, both in shape and in yield, within better than 5\%. More details on the experimental apparatus and data analysis will be given in a forthcoming extended paper; for now see Refs. [15,17].

Figure 1 shows the opposite-sign, background, and signal dimuon mass spectra, integrated over all collision centralities. After subtracting the combinatorial background and the signal fake matches, the resulting net spectrum contains about 360000 muon pairs in the mass range of the figure, roughly $50 \%$ of the total available statistics. The average charged-particle multiplicity density measured by the vertex tracker is $d N_{\mathrm{ch}} / d \eta=120$, the average signal-to-background ratio is $1 / 7$. For the first time in nuclear collisions, the vector mesons $\omega$ and $\phi$ are completely resolved in the dilepton channel; even the $\eta \rightarrow$ $\mu \mu$ decay is seen. The mass resolution at the $\omega$ is $20 \mathrm{MeV} / c^{2}$. The subsequent analysis is done in four classes of collision centrality defined through the charged-particle multiplicity density: peripheral (4-30), semiperipheral (30-110), semicentral (110-170), and central (170-240). The signal-to-background ratios associated with the individual classes are $2,1 / 3,1 / 8$, and $1 / 11$, respectively. 


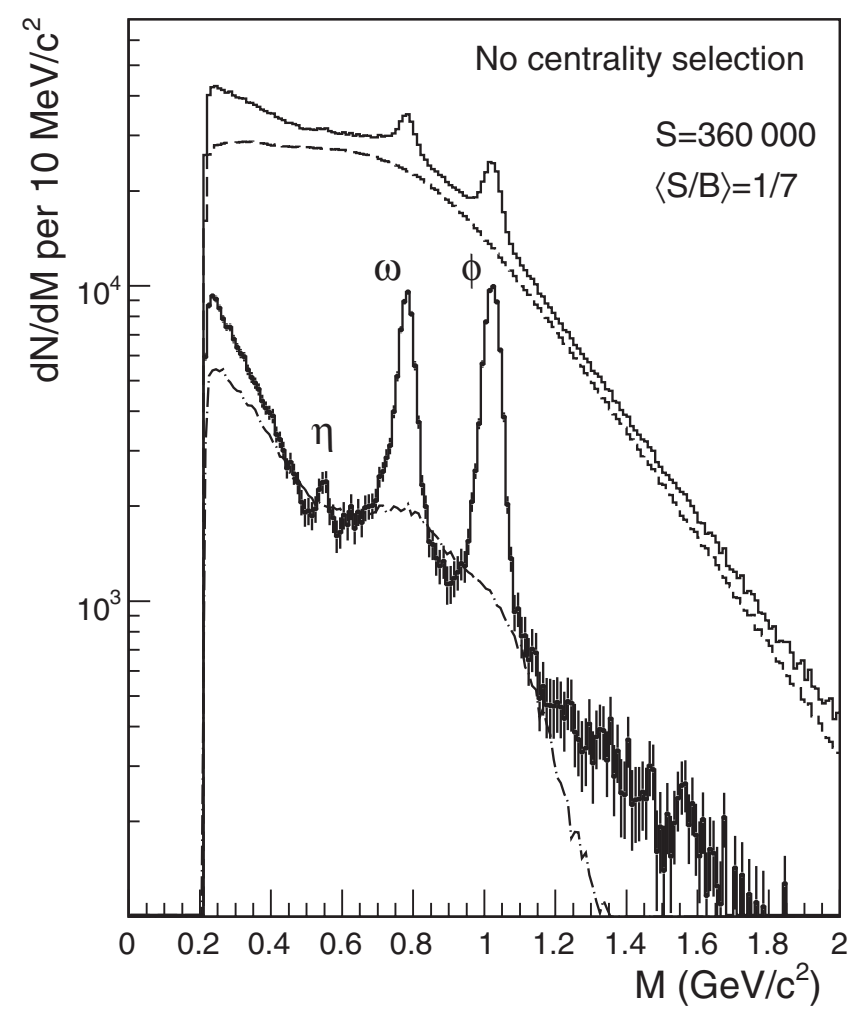

FIG. 1. Mass spectra of the opposite-sign dimuons (upper histogram), combinatorial background (dashed line), signal fake matches (dashed-dotted line), and resulting signal (histogram with error bars).

The peripheral data can be described by the expected electromagnetic decays of the neutral mesons [18]. Muon pairs from resonance $(\eta, \rho, \omega, \phi)$ and Dalitz $\left(\eta, \eta^{\prime}, \omega\right)$ decays were simulated using the improved hadron decay generator GENESIS [19] as the input and GEANT for transport through the detectors. Four free parameters (apart from the overall normalization) were used in the fit of this "hadron decay cocktail" to the peripheral data: the cross section ratios $\eta / \omega, \rho / \omega$ and $\phi / \omega$, and the level of D meson pair decays; the ratio $\eta^{\prime} / \eta$ was kept fixed at 0.12 $[10,19]$. The fits were independently done in 3 bins of dimuon transverse momentum: $p_{T}<0.5,0.5<p_{T}<1$, and $p_{T}>1 \mathrm{GeV} / c$. The particle ratios obtained from the fits without $p_{T}$ selection, corrected for acceptance and extrapolated to full phase space are $\eta / \omega=0.88 \pm 0.03$, $\phi / \omega=0.094 \pm 0.004$, and $\rho / \omega=1.62 \pm 0.10$. The errors quoted are purely statistical; the systematic errors are estimated to be of order $10 \%$, dominated by uncertainties of the branching ratios (using the much more accurate value for $\omega \rightarrow e^{+} e^{-}$instead of $\mu^{+} \mu^{-}$with the assumption of $e \mu$ universality). The value for $\eta / \omega$ agrees, within errors, with the literature average of $0.82 \pm 0.11$ for $p$ - $p$, $p$-Be [10]. The value for $\phi / \omega$ is subject to some $\phi$-enhancement already in peripheral nuclear collisions and can therefore not be directly compared to $p$ - $p$ values. Within $10 \%$ these two ratios do not depend on the pair $p_{T}$. In contrast, the particle ratio $\rho / \omega$ monotonically decreases with $p_{T}$ [18], reaching $1.20 \pm 0.09$ at $p_{T}>1 \mathrm{GeV} / c$, where it agrees with the literature average of $1.0 \pm 0.15$ for $p-p, p$-Be [10] within errors. This suggests that some $\pi \pi$ annihilation, enhancing the yield of the low- $p_{T} \rho$, contributes already in peripheral collisions (see below). The level of charm decays obtained from the fits is essentially determined by the measured yield in the mass interval $1.2<M<1.4 \mathrm{GeV} / c^{2}$. The results altogether, and, in particular, those on the $p_{T}$ independence of $\eta / \omega$ and $\phi / \omega$, indicate that the acceptance of NA60 both in mass and $p_{T}$, including the critical low-mass ( $\eta$-Dalitz), low- $p_{T}$ region $(<0.5 \mathrm{GeV} / c)$, is reasonably well understood. Apart from the sources discussed, no further input was required to describe the peripheral data.

In the more central bins, the data can no longer be described on the basis of the standard hadron decay cocktail alone, but is indicative of the existence of an excess yield. Since the particle ratios are expected to be different from the peripheral data, global fits to the more central data are bound to bias both the extracted cocktail parameters and an excess with a priori unknown characteristics. We have therefore used a novel procedure, made possible by the high data quality; it is illustrated in Fig. 2. The excess is isolated by subtracting the cocktail, without the $\rho$, from the data. The cocktail is fixed, separately for the major sources and in each centrality bin, by a "conservative" approach. The yields of the narrow vector mesons $\omega$ and $\phi$ are fixed

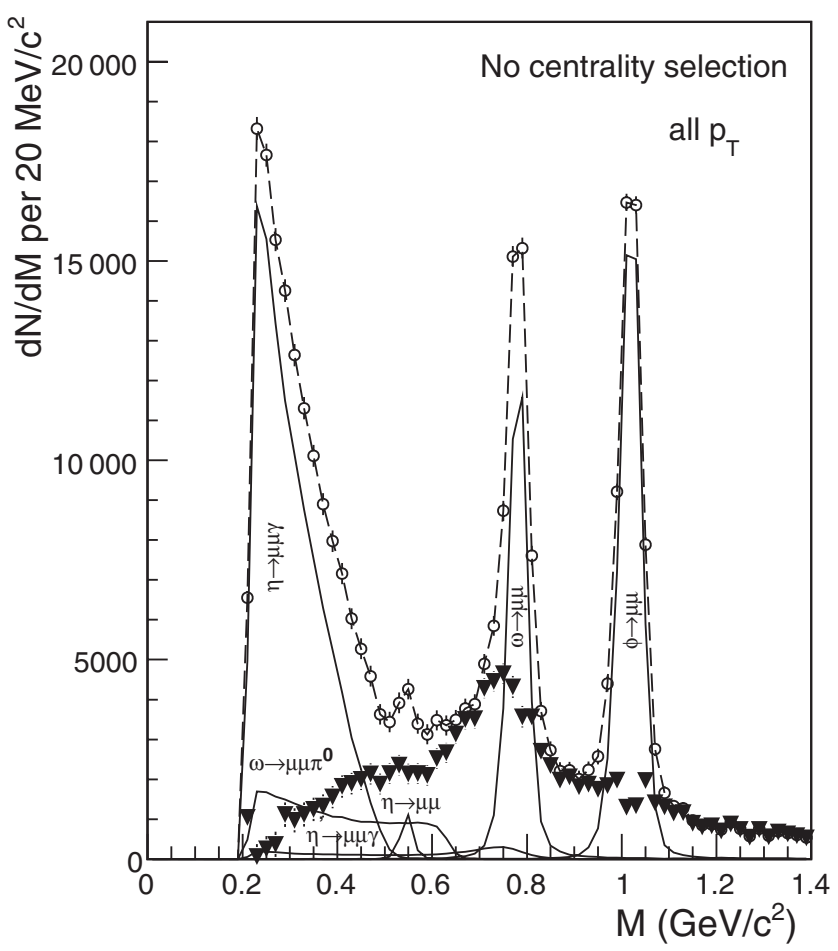

FIG. 2. Isolation of an excess above the electromagnetic decays of a conservative hadron decay cocktail (see text). Total data (open circles), individual cocktail sources (solid line), difference data (thick triangles), sum of cocktail sources and difference data (dashed line). 
so as to get, after subtraction, a smooth underlying continuum. For the $\eta$, an upper limit is defined by "saturating" the measured data in the region close to $0.2 \mathrm{GeV} / c^{2}$; this implies the excess to vanish at very low mass, by construction. The $\eta$ resonance and $\omega$ Dalitz decays are now bound as well; $\eta^{\prime} / \eta$ is fixed as before. The cocktail $\rho$ (only required in Figs. 3 and 4, for illustration purposes) is bound by the ratio $\rho / \omega=1.2$, found at high $p_{T}(>1.6 \mathrm{GeV} / c)$ for all centralities. The accuracy in the determination of the $\omega$ and $\phi$ yields by this subtraction procedure is on the level of $1-2 \%$, due to the remarkable local sensitivity, and not much worse for the $\eta$. The qualitative features of the resulting difference spectrum are robust towards yield changes even on the level of $10 \%$, again because the consequences of such changes are highly localized.

The excess mass spectra for all 4 multiplicity bins, resulting from subtraction of the conservative hadron decay cocktail from the measured data, are shown in Fig. 3. The cocktail $\rho$ and the level of charm decays, found in the 3 upper centrality bins to be about $1 / 3$ of the measured yield in the mass interval $1.2<M<1.4 \mathrm{GeV} / c^{2}$ [15], are shown for comparison. The qualitative features of the spectra are striking: a peaked structure is seen in all cases, broadening strongly with centrality, but remaining essentially centered around the position of the nominal $\rho$ pole. At the same time, the total yield increases relative to the cocktail $\rho$, their ratio reaching values above 4 for $M<$ $0.9 \mathrm{GeV} / c^{2}$ in the most central bin. Such values are consistent with the results found by CERES [12], if the latter are also referred to the cocktail $\rho$ and rescaled according to the different multiplicity density. The errors shown are purely statistical. The dominant sources of systematic errors are connected to the uncertainties in the levels of the combinatorial background (1\%) and fake matches (5\%). On the basis of these values and the signal-to-background ratios, the systematic errors in the broad continuum region $0.4<M<0.6$ and $0.8<M<1.0 \mathrm{GeV} / c^{2}$ are estimated
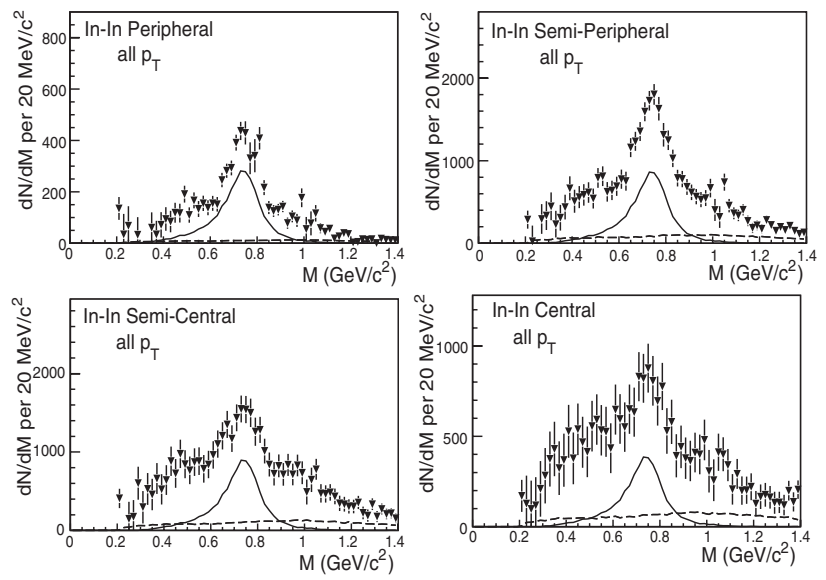

FIG. 3. Excess mass spectra of dimuons. The cocktail $\rho$ (solid lines) and the level of uncorrelated charm decays (dashed lines) are shown for comparison. The errors are purely statistical; see text for systematic errors. to be about $3 \%, 12 \%, 25 \%$, and $25 \%$ in the 4 centrality bins, from peripheral to central. Uncertainties associated with the hadron decay cocktail and its subtraction including branching ratios, transition form factors and helicity distributions have been estimated to be around $15 \%$. Since the background causing the dominant errors is essentially flat, the $\rho$-like structure above the continuum is much more robust.

The qualitative features of the mass spectra in Fig. 3 are consistent with an interpretation of the excess as dominantly due to $\pi \pi$ annihilation. Among the many different theoretical predictions for the properties of the intermediate $\rho$ mentioned in the introduction, only two have been brought to a level suitable for a quantitative comparison to the data: the broadening scenario of Refs. $[4,5]$ and the moving-mass scenario related to Refs. [6,7]. Both are evaluated for In-In at $d N_{\mathrm{ch}} / d \eta=140$ within the same fireball evolution, taking explicit account of temperature as well as of baryon density [20]. In Fig. 4, these predictions (as well as the unmodified $\rho$ ) are confronted with the data for the semicentral bin (same charged-particle rapidity density). Note that the integrals of the theoretical spectra are normalized to the data in the mass interval $M<$ $0.9 \mathrm{GeV} / c^{2}$. The unmodified $\rho$ is clearly ruled out. The specific moving-mass scenario plotted here, which fit the CERES data [5,12], is also ruled out, showing the much improved discrimination power of the present data. The broadening scenario appears more realistic. However, the nearly symmetrical broadening around the $\rho$ pole seen in the data is not reproduced by this model. The remaining

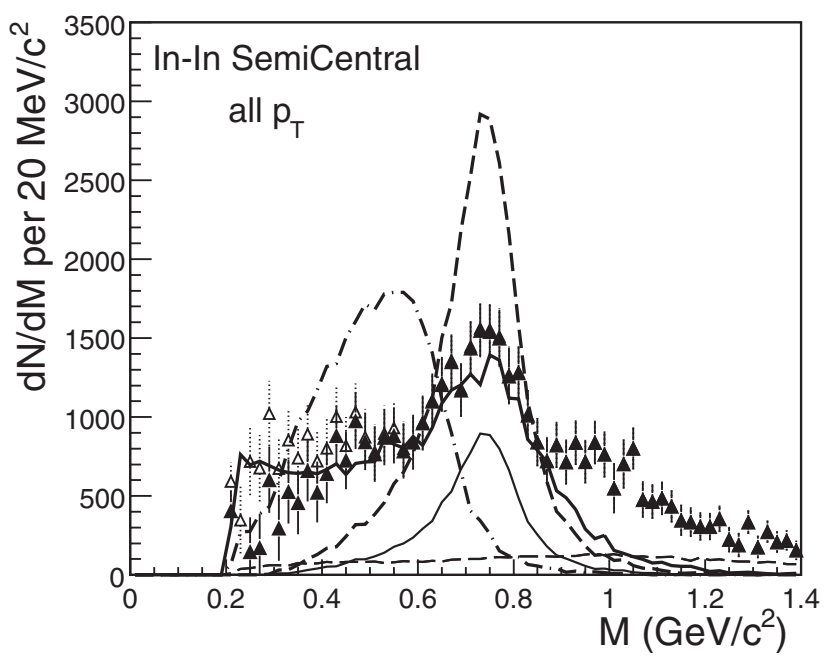

FIG. 4. Comparison of the excess mass spectrum for the semicentral bin to model predictions, made for In-In at $d N_{\mathrm{ch}} / d \eta=$ 140. Cocktail $\rho$ (thin solid line), unmodified $\rho$ (dashed line), inmedium broadening $\rho[4,5]$ (thick solid line), in-medium moving $\rho$ related to $[6,7]$ (dashed-dotted line). The errors are purely statistical. The systematic errors of the continuum are about $25 \%$ (see text). The open data points show the difference spectrum resulting from a decrease of the $\eta$ yield by $10 \%$ (which should also be viewed as a systematic error). 
excess at $M>0.9 \mathrm{GeV} / c^{2}$ may well be related to the prompt dimuon excess found by NA60 in the intermediate mass region [15]. Processes other than $2 \pi$, i.e., $4 \pi \ldots$, could possibly account for the region $M>0.9 \mathrm{GeV} / c^{2}$ [21].

The data shown in this Letter have not been corrected for the mass- and $p_{T}$-dependent acceptance of the NA60 setup. The theoretical calculations shown in Fig. 4 were therefore also propagated through the acceptance filter to allow for fair comparison with the data. It is interesting to note that, by coincidence, the propagation of theoretical calculations based on a white spectral function (e.g., $q \bar{q}$ annihilation [20]) yields a mass spectrum flat within $10 \%$ up to about $1 \mathrm{GeV} / c^{2}$, without any bump structure. In other words, the always existing steep rise of the theoretical input at low masses, due to the photon propagator and a Boltzmann-like factor [4-7], is just about compensated by the falling acceptance in this region as long as no $p_{T}$ cut is applied. The data and model predictions shown in Figs. 3 and 4 can therefore be interpreted as spectral functions of the $\rho$, averaged over momenta and the complete space-time evolution of the fireball. The flat part of the measured spectra may thus reflect the early history close to the QCD boundary with a nearly divergent width, while the narrow peak on top may just be due to the late part close to the thermal freezeout, approaching the nominal width.

We conclude, in quite general terms and independently of any comparison to theoretical modeling, that the $\rho$ primarily broadens in In-In collisions, but does not show any noticeable shift in mass. More detailed work including precise $p_{T}$ dependences is under way to consolidate these findings.

[1] R. D. Pisarski, Phys. Lett. 110B, 155 (1982).
[2] C. A. Dominguez, M. Loewe, and J. C. Rojas, Z. Phys. C 59, 63 (1993).

[3] R. D. Pisarski, Phys. Rev. D 52, R3773 (1995).

[4] G. Chanfray, R. Rapp, and J. Wambach, Phys. Rev. Lett. 76, 368 (1996); R. Rapp, G. Chanfray, and J. Wambach, Nucl. Phys. A617, 472 (1997).

[5] R. Rapp and J. Wambach, Adv. Nucl. Phys. 25, 1 (2000).

[6] G. E. Brown and M. Rho, Phys. Rev. Lett. 66, 2720 (1991); G. Q. Li, C. M. Ko, and G. E. Brown, Phys. Rev. Lett. 75, 4007 (1995).

[7] G. E. Brown and M. Rho, Phys. Rep. 363, 85 (2002).

[8] T. Hatsuda and S. H. Lee, Phys. Rev. C 46, R34 (1992).

[9] H. Leutwyler and A. V. Smilga, Nucl. Phys. B342, 302 (1990).

[10] G. Agakichiev et al. (CERES Collaboration), Eur. Phys. J. C 4, 231 (1998).

[11] G. Agakichiev et al. (CERES Collaboration), Phys. Rev. Lett. 75, 1272 (1995).

[12] G. Agakichiev et al. (CERES Collaboration), Phys. Lett. B 422, 405 (1998); B. Lenkeit et al., Nucl. Phys. A661, 23c (1999); G. Agakichiev et al., Eur. Phys. J. C 41, 475 (2005).

[13] G. Usai et al. (NA60 Collaboration), Eur. Phys. J. C 43, 415 (2005).

[14] M. Keil et al., Nucl. Instrum. Methods Phys. Res., Sect. A 539, 137 (2005); 546, 448 (2005).

[15] R. Shahoyan et al. (NA60 Collaboration), Eur. Phys. J. C 43, 209 (2005); Quark Matter, Budapest, 2005.

[16] M. Floris et al. (NA60 Collaboration), ISMD05, Kromeriz, 2005.

[17] A. David, Ph.D. Thesis, Instituto Superior Técnico, Lisbon, 2006.

[18] E. Scomparin et al. (NA60 Collaboration), Quark Matter, Budapest, 2005.

[19] S. Damjanovic, A. De Falco, and H. Wöhri (NA60 Collaboration), NA60 Internal Note 2005-1.

[20] R. Rapp (private communication).

[21] G. Q. Li and C. Gale, Phys. Rev. Lett. 81, 1572 (1998); Phys. Rev. C 58, 2914 (1998). 